\title{
Acute myocardial infarction incidence and hospital mortality: routinely collected national data versus linkage of national registers
}

\author{
Huberdina L. Koek · Jan W. P. F. Kardaun · Evelien Gevers • \\ Agnes de Bruin · Joannes B. Reitsma · Diederick E. Grobbee • \\ Michiel L. Bots
}

Received: 16 May 2007/ Accepted: 26 July 2007/Published online: 8 September 2007

(C) Springer Science+Business Media B.V. 2007

\begin{abstract}
Background and Objective To compare levels of and trends in incidence and hospital mortality of first acute myocardial infarction (AMI) based on routinely collected hospital morbidity data and on linked registers. Cases taken from routine hospital data are a mix of patients with recurrent and first events, and double counting occurs when cases are admitted for an event several times during 1 year. By linkage of registers, recurrent events and double counts can be excluded.

Study Design and Setting In 1995 and 2000, 28,733 and 25,864 admissions for AMI were registered in the Dutch national hospital discharge register. Linkage with the population register yielded 21,565 patients with a first AMI in 1995 and 20,414 in 2000.

Results In 1995 and 2000, the incidence based on the hospital register was higher than based on the linked registers in men (22\% and $23 \%$ higher) and women (18\%
\end{abstract}

Electronic supplementary material The online version of this article (doi:10.1007/s10654-007-9174-6) contains supplementary material, which is available to authorized users.

H. L. Koek · D. E. Grobbee · M. L. Bots $(\bowtie)$

Julius Center for Health Sciences and Primary Care, University

Medical Center, Utrecht, HPN Str. 06.131, P.O. Box 85.060,

3508 AB Utrecht, The Netherlands

e-mail: m.l.bots@umcutrecht.nl

J. W. P. F. Kardaun · A. de Bruin

Statistics Netherlands, Voorburg, The Netherlands

E. Gevers

Prismant, Utrecht, The Netherlands

J. B. Reitsma

Department of Clinical Epidemiology and Biostatistics,

Academic Medical Center, Amsterdam, The Netherlands and $20 \%$ higher). In both years, hospital mortality based on the hospital register and on linked registers was similar. The decline in incidence between 1995 and 2000 was comparable whether based on standard hospital register data or linked data (18\% and $20 \%$ in men, $15 \%$ and $17 \%$ in women). Similarly, the decline in hospital mortality was comparable using either approach $(11 \%$ and $9 \%$ in both men and women).

Conclusion Although the incidence based on routine hospital data overestimates the actual incidence of first AMI based on linked registers, hospital mortality and trends in incidence and hospital mortality are not changed by excluding recurrent events and double counts. Since trends in incidence and hospital mortality of AMI are often based on national routinely collected data, it is reassuring that our results indicate that findings from such studies are indeed valid and not biased because of recurrent events and double counts.

Keywords Acute myocardial infarction . Medical record linkage · Registries · Incidence · Epidemiology $\cdot$ Hospital admissions

\section{Introduction}

Mortality from coronary heart disease, in particular from acute myocardial infarction (AMI), has decreased in many Western countries during the last decades [1]. A decrease in age- and gender-adjusted AMI mortality, assuming a constant quality of diagnosis, is a consequence of either a decrease in incidence, case-fatality or recurrence risk, or a combination of these. Hospital-based registers are often used for surveillance of the morbidity and hospital mortality of AMI [2]. In the Netherlands, the national hospital 
discharge register has traditionally been used to provide estimates of (trends in) incidence and hospital mortality of AMI [3]. However, in this register, like in many others, a new record is created for each hospital admission. As a consequence, admissions taken from the hospital register will include double counts from patients if they are transferred to a second hospital or if they are admitted for the same event several times during 1 year. Furthermore, patients taken from the hospital register from 1 year include a mix of patients with recurrent events (presence of an event in preceding years) and first events (absence of events in preceding years). Tracking individuals over time based on information from the hospital register only is difficult when a unique personal identifier is absent in the hospital register. The effect of both double counting and admixture of first and recurrent events in nationwide registers on (trends) in incidence or hospital mortality has not been well assessed [4, 5]. For the Netherlands, this effect could only be estimated from comparison with results from regional cohort studies, as nationwide estimates of incidence and hospital mortality of first AMI were not available. Furthermore, it has been argued that statistics from routine data could not be used for providing reliable information on (trends in) incidence and hospital mortality. After we recently showed that hospitalized patients in the Netherlands can be followed longitudinally within the national hospital discharge register in a valid way by using information from the Dutch population register [6], we set out to compare the nationwide (trends in) incidence and hospital mortality of first hospitalized AMI based on routinely collected data in the hospital register (double counts and recurrent events included) and based on linkage of the hospital register with the population register (double counts and recurrent events excluded).

\section{Methods}

Data sources

Data on hospital admissions were retrieved from the national hospital discharge register. Since 1986, all general and academic hospitals and most single specialty hospitals participate in the hospital register. There are no private hospitals in the Netherlands that treat patients with AMI. For each hospital admission a new record is created in the hospital register, including the following information: date of birth, gender, numeric part of postal code (since 1991), hospital-specific patient identification code, type of hospital, admission date and principal diagnosis of the admission. The principal diagnosis is determined at discharge and coded using the ninth revision of the International Classification of Diseases (ICD-9-CM) [7].
As the hospital register does not contain a unique personal identifier, we tracked individuals over time within the hospital register by using information from the Dutch population register. This database contains information on all registered persons living in the Netherlands, including date of birth, gender, current address, postal code and nationality. Patients registered in the hospital register were identified in the population register using linkage variables 'date of birth', 'gender' and 'numeric part of postal code'. When patients moved, their hospital admissions were recognized by using the new postal code registered in the population register.

Recently, the validity of the registries and linkage methods was studied. In a random sample of hospital admissions, $99 \%$ of the personal, admission and discharge data and $84 \%$ of the principal diagnoses (validated through medical record review by medical specialists) were correctly registered [8]. In a random sample of the population register, over $97 \%$ of the addresses were shown to be correctly registered [9]. Furthermore, over 97\% of the uniquely linked hospital admissions resulting from linkage of the hospital register with the population register were shown to be correctly linked [9].

These results are similar to most of the studies that reported on the validity of AMI events in hospital and population based registries [10-13].

All analyses were performed at Statistics Netherlands in agreement with privacy legislation in the Netherlands [6].

\section{Cohort enrolment from the hospital register}

The hospital register comprises information based on all admissions in the Netherlands of the entire Dutch population, including double counts, first and recurrent admissions for AMI, and including AMI admissions of non-residents. In the hospital register, 28,733 and 25,864 hospital admissions with a principal diagnosis AMI (ICD-9-CM [7] code 410 and subcategories) were registered in 1995 and 2000.

\section{Cohort enrolment from linked registers}

After linkage with the population register using linkage variables 'date of birth', 'gender' and 'numerical part of postal code', 25,142 and 22,470 admissions came from patients with a unique combination of linkage variables in the population register ( $88 \%$ and $87 \%$, respectively). Thus, each remaining admission linked to only one unique individual in the population register (one unique individual in the Netherlands). Admissions linking with more than one person (e.g., administrative twins; two persons with the 
same date of birth, gender and numeric part of postal code registered in the population register) or with no person at all (e.g., non-residents or administrative errors) in the population register were excluded. Selection of the first admission per person of all subsequent admissions of a person occurring during 1995 and 2000 yielded 23,172 patients in 1995 and 20,414 patients in 2000. Thus, 1,970 double counts had occurred in 1995 (8\%) and 2,056 in $2000(9 \%)$. Information on admissions in previous years of the patients in 1995 was obtained by selecting all hospital admissions registered in the hospital register with principal diagnosis AMI in the period 1991-1995. These admissions were linked to the cohort of 23,172 patients with linkage variables 'date of birth', 'gender' and one or both of the variables 'numerical part of postal code' and 'hospitalspecific patient identification code'. Linkage with the population register was not possible, since this register started in October 1994. Subjects who linked in this process were patients with previous hospital admissions for AMI (recurrent AMI patients) and were excluded (1,607 patients $(7 \%)$ ). This resulted in the final cohort of 1995 consisting of 21,565 patients. Information on hospital admissions in previous years of the patients in 2000 was obtained by linking of the hospital register of 1995-2000 to the population register with linkage variables 'date of birth', 'gender' and 'numerical part of postal code'. All uniquely linked admissions with principal diagnosis AMI were selected and linked to the cohort of 20,414 patients. Patients with previous hospital admissions for AMI (recurrent AMI patients) were excluded (1,356 patients (7\%)). This resulted in the final cohort of 2000 consisting of 19,058 patients.

Thus the linked register comprises information for only part of the Dutch population (i.e., those that were unique on date of birth, gender, and postal code), and does not include double counts, and recurrent AMI admissions.

\section{Data analysis}

The incidence and hospital mortality of AMI (with 95\% confidence interval $(95 \% \mathrm{CI})$ ) based on the hospital register (hospital-based) and on linked registers (linkage-based) was computed by year, age and gender. We compared the hospital-based incidence and hospital mortality to the linkage-based incidence and hospital mortality by calculating incidence rate differences or ratios and risk differences or ratios (with $95 \%$ CIs) by age and gender. Trends in incidence and hospital mortality were obtained by calculating incidence rate or risk differences and incidence rate or risk ratios (with $95 \%$ CIs) by age and gender. Incidence rate differences and ratios were based on the Poisson model, while risk differences and ratios were based on the binomial model [14]. Pooled age-adjusted incidence rate differences or ratios and risk differences or ratios (with 95\% CIs) were calculated according to the Mantel Haenszel method [15].

\section{Results}

In both 1995 and 2000, the gender and age distribution of the cohort based on the hospital register was comparable to the cohort based on linked registers. In 1995 and 2000, two-thirds comprised men. The mean age in 1995 was 63.7 years in men and 71.5 years in women based on the hospital register. This was 64.3 and 71.9 years, respectively, based on linked registers. In 2000, the mean age based on the hospital register was 63.6 years in men and 70.9 years in women. Based on linked registers, this was 64.2 and 71.6 years, respectively.

In men, the (adjusted) hospital-based incidence was 47 per 100,000 person-years or $22 \%$ (95\% CI 19-25\%) higher than the (adjusted) linkage-based incidence in 1995 and 43 per 100,000 person-years or $23 \%$ (95\% CI 20-26\%) higher in 2000 (Table 1). Age-specific ( $\geq 30$ years) absolute and relative differences ranged from 9-217 per 100,000 personyears or $20-28 \%$ in 1995 and from $7-220$ per 100,000 person-years or $22-25 \%$ in 2000 . The (adjusted) hospitalbased incidence was also higher than the (adjusted) linkage-based incidence in women in 1995 (19 per 100,000 person-years or $18 \%$; 95\% CI 15-22\% higher) and 2000 (18 per 100,000 person-years or $20 \%$; $95 \%$ CI $16-24 \%$ higher). Age-specific ( $\geq 30$ years) absolute and relative differences varied from 3-103 per 100,000 person-years or $13-20 \%$ in 1995 and from $1-116$ per 100,000 person-years or $14-33 \%$ in 2000 .

The hospital-based hospital mortality was similar to the linkage-based hospital mortality in men in 1995 (adjusted risk ratio (RR) 1.01; 95\% CI 0.95-1.07) and 2000 (adjusted RR 1.00; 95\% CI 0.94-1.07) and in women in 1995 (adjusted RR 0.98; 95\% CI 0.92-1.05) and 2000 (adjusted RR 0.99; 95\% CI 0.93-1.06) (Table 2). Also within the age groups, no significant differences between the hospitalbased and the linkage-based hospital mortality were revealed.

From 1995 to 2000, the hospital-based decline in incidence was similar to the linkage-based decline (Table 3). In men, the (adjusted) hospital-based incidence declined by 48 per 100,000 person-years or $18 \%$ (95\% CI 17-20\%) and the linkage-based incidence declined by 46 per 100,000 person-years or $20 \%$ (95\% CI 18-22\%). In women, the (adjusted) hospital-based incidence declined by 18 per 100,000 person-years or $15 \%$ (95\% CI 13-18\%) and the (adjusted) linkage-based incidence declined by 18 per 100,000 persons per year or $17 \%$ (95\% CI 14-19\%). The 


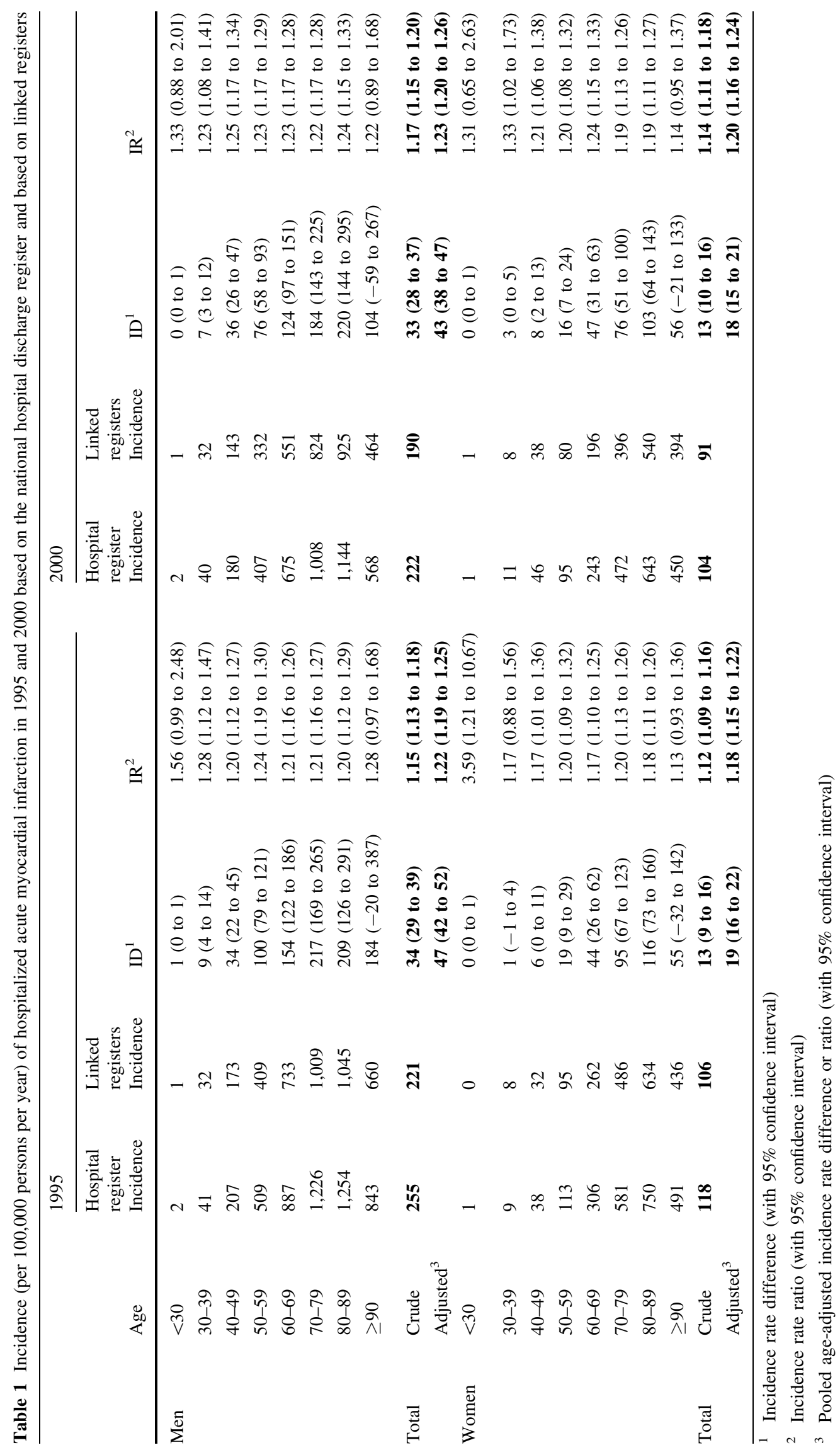




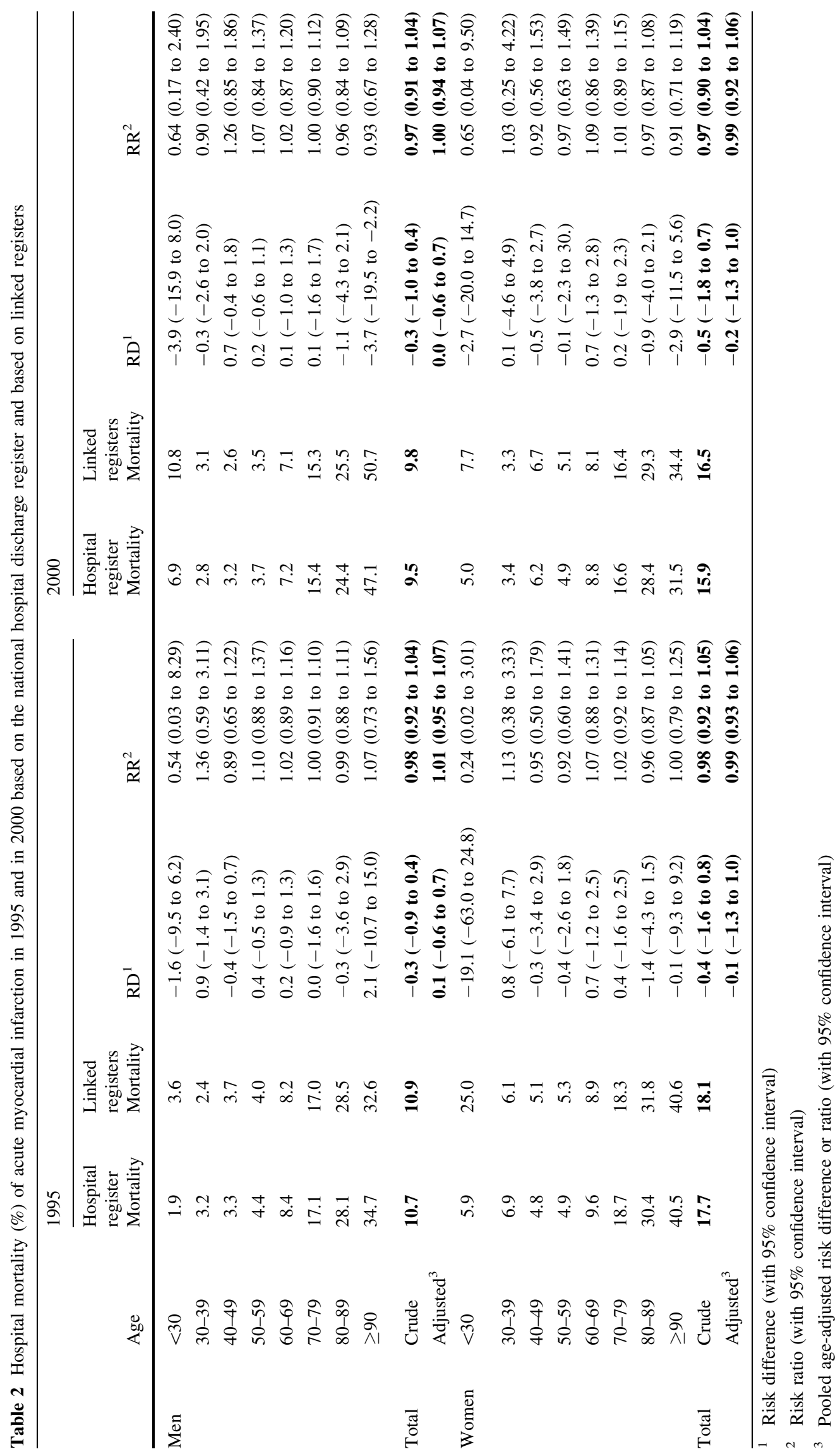




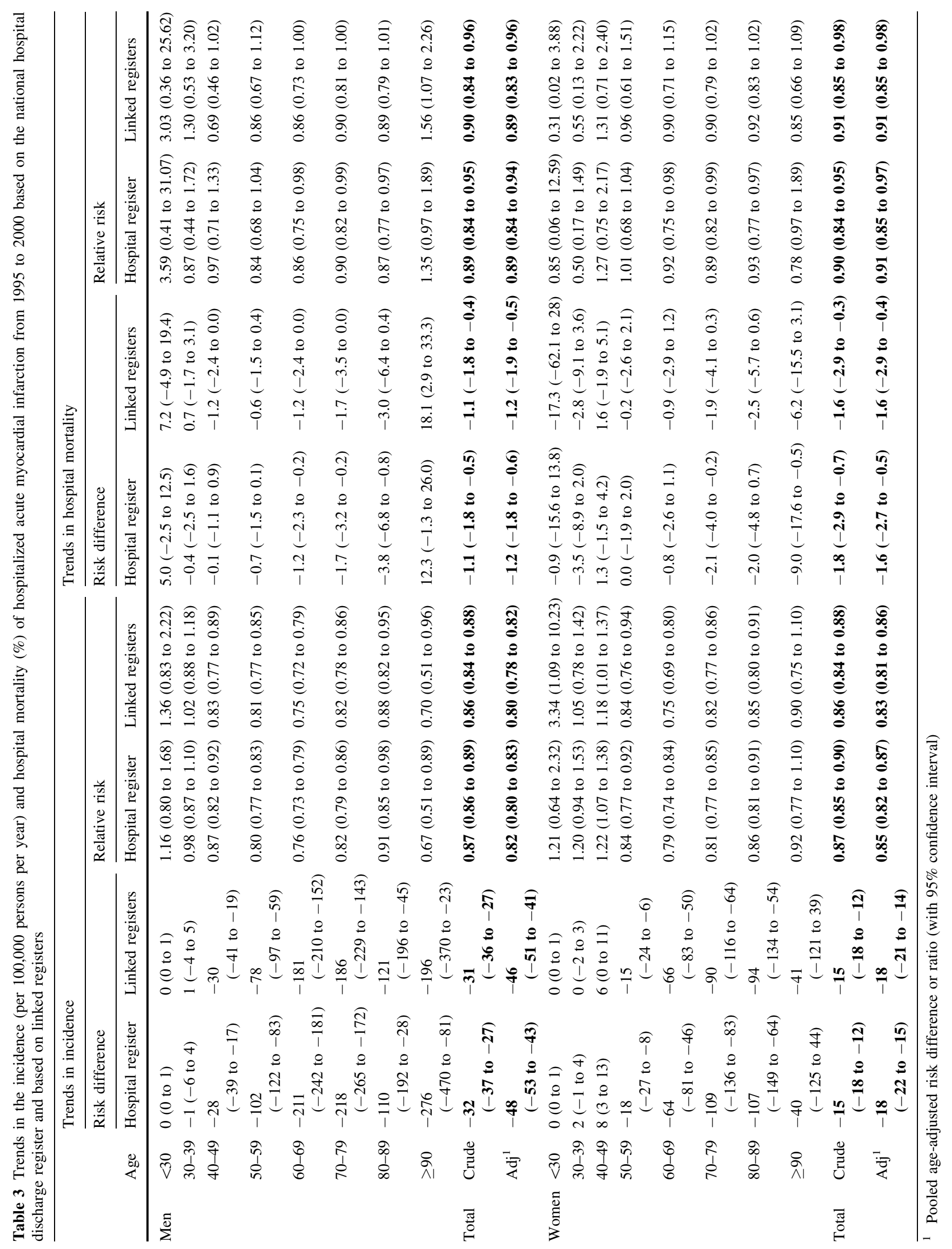


age-specific relative changes in hospital-based and linkagebased incidence were largely comparable.

The (adjusted) hospital-based decline in hospital mortality from 1995 to 2000 was similar to the (adjusted) linkage-based decline (Table 3). In men, hospital mortality declined absolutely by $1 \%$ and relatively by $11 \%$ based on both the hospital register and linked registers. In women, the absolute and relative decline was $2 \%$ and $9 \%$, respectively, based on both the hospital register and linked registers. The age-specific relative changes in hospitalbased and linkage-based hospital mortality were largely similar.

\section{Discussion}

We combined data from the national hospital discharge register with data from the population register to determine the (trends in) incidence and hospital mortality of first hospitalized AMI (double counts and recurrent AMI cases excluded) and compared the outcomes with the incidence and hospital mortality based on routinely collected data in the hospital register (double counts and recurrent AMI cases included). The incidence based on the hospital register was considerably and significantly higher than the incidence based on linked registers, whereas hospital mortality and trends in incidence and hospital mortality were identical using either approach.

Although we were able to estimate the incidence and hospital mortality of first AMI by linkage of the hospital register with the population register, some aspects of this method should be discussed. First, non-unique persons in the population (register) were excluded from the study population in the linked registry data. If this exclusion produced systematic differences between the linked registry population and the clinically relevant population (i.e., the total Dutch population), it might have affected the incidence estimate in the linked registry to some extent (e.g., an overestimation of incidence resulting from a higher mean age of the study population). A pilot study suggested that non-uniqueness relates to large cities, foreign origin and age [6]. The differences between unique and non-unique persons, however, were small [9] and apply to both 1995 and 2000. Second, information on previous admissions was limited to maximal 5 years for the patients (as the numeric part of the postal code is registered in the hospital register since 1991). Therefore, it seems likely that some "first" AMI patients actually were recurrent AMI patients. However, it has been estimated that most (95\%) of recurrent events occur within 5 years. $[4,16]$ Third, the outcome measures in the present study were incidence and hospital mortality. Mortality after discharge from hospital was not considered, since this outcome is not registered in the hospital register. Differences in mortality after discharge between patients with a first or a recurrent AMI can only be studied by linkage of national registers (i.e., the hospital register with the population register and the cause of death statistics). A final aspect that needs to be addressed is the generalizability of our findings. The results might differ if a change over time occurs in double-count or readmission fractions. Results might also differ for other diseases than AMI or for specific groups of patients (e.g., non-native patients), hospitals or regions. Such differences will not be apparent from routinely collected data. Since trends in incidence and hospital mortality are often based on national routinely collected data, generalization of our findings would be of great relevance.

It has been be argued that routine statistics can not be used for providing information on (trends in) incidence and hospital mortality, because of double counting of cases and admixture of first and recurrent events. In order to prevent erroneous inclusion of prevalent cases (recurrent events) that have had a previous hospitalization for AMI prior to the study period generally an clearance period is employed to overcome overestimation of the incidence [16]. For myocardial infarction, it has been shown that a clearance period of 13 years should be taken into account to completely overcome inclusion of prevalent cases. In our study we used a 5-year clearance period because of logistical reasons. This however would suggest that in our incidence estimate around $5 \%$ of the subjects should be considered as recurrent AMI-patient rather that first ever AMI patient [16]. Although this does affect the absolute incidence estimate, it may not affect trends in incidence and case fatality, assuming that the erroneous inclusion of prevalent cases occurs at both time windows Indeed this has been shown in a Danish study where the incidence based on the number of AMI-patients without an admission for AMI in the previous year overestimated the incidence based on the number of AMI-patients without (an admission for) AMI in the previous 14 years by $27 \%$ in men and $16 \%$ in women, but trends reflected trends in true incidence with reasonable accuracy [4]. With respect to double count, in eight states of the USA, it was estimated that double counting of patients resulted in an overestimation of the true incidence of hospitalized AMI and an underestimation of the true hospital mortality. In this study, double counts were defined as readmissions for AMI within 7 days and thought to result from transfer to a second hospital. Correction for double counting revealed a 10-15\% lower incidence and a $12 \%$ higher hospital mortality [5]. Despite aspects regarding double counts and recurrent events, there have been several consistent reports from different countries using national registries to study trends in case fatality, incidence and survival [17-21]. These time trends indicated a decline in incidence of myocardial infarction 
and in case fatality after AMI. In the present study, we found a significant decline in both incidence and hospital mortality of first AMI between 1995 and 2000. These declines in incidence and hospital mortality appear to mainly reflect declines in first events, as trends were not altered when recurrent cases were excluded from the data, and thus are best explained by advances in primary prevention and acute management of AMI.

Overall, our results based on deterministic linkages of sources using gender, date of birth and postal code, are in line with earlier reports from other studies, where linkage was performed using unique identification numbers.

In conclusion, our study shows that the incidence based on routinely collected data in the national hospital discharge register overestimates the actual incidence of first AMI based on linked national registers by least $22 \%$ in men and $18 \%$ in women. Yet, the hospital mortality based on the hospital register accurately reflects the actual hospital mortality of first AMI. Furthermore, trends in incidence and hospital mortality based on the hospital register are not changed when double counts and recurrent cases were excluded. Since trends in incidence and hospital mortality of AMI are often based on national routinely collected data, it is reassuring that our results indicate that findings from such studies are indeed valid and not biased because of recurrent events and double counts.

Acknowledgements This study was supported by a grant from the Netherlands Heart Foundation (grant number 31632501). The study was part of the project 'Cardiovascular disease in the Netherlands: figures and facts' of the Netherlands Heart Foundation.

\section{References}

1. Levi F, Lucchini F, Negri E, et al. Trends in mortality from cardiovascular and cerebrovascular diseases in Europe and other areas of the world. Heart 2002;88:119-24.

2. Fang J, Alderman MH. Dissociation of hospitalization and mortality trends for myocardial infarction in the United States from 1988 to 1997 . Am J Med 2002;113:208-14.

3. Koek HL, Grobbee DE, Bots ML. Trends in cardiovascular morbidity and mortality in the Netherlands, 1980-2000.Ned Tijdschr Geneeskd 2004;148:27-32 [in Dutch].

4. Osler M, Rostgaard K, Sørensen TIA, et al. The effect of recurrent events on register-based estimates of level and trends in incidence of acute myocardial infarction. J Clin Epidemiol 1999;52:595-600.

5. Westfall JM, McGloin J. Impact of double counting and transfer bias on estimated rates and outcomes of acute myocardial infarction. Med Care 2001;39:459-68.

6. Reitsma JB, Kardaun JWPF, Gevers E, et al. Possibilities for anonymous follow-up studies of patients in Dutch national medical registrations using the Municipal Population Register: a pilot study. Ned Tijdschr Geneesk 2003;147:2286-90 [in Dutch].

7. The International Statistical Classification of Diseases, Injuries and Causes of Death. Ninth Revision. Clinical Modification. Washington DC: U.S. Department of Health and Human Services 1979.

8. Paas GRA, Veenhuizen KCW. Research on the validity of the LMR. Utrecht: Prismant, 2002 [in Dutch].

9. De Bruin A, Kardaun JWPF, Gast A, et al. Record linkage of hospital discharge register with population register: experiences at Statistics Netherlands. Stat J UN Econ Comm Eur 2004;21:23-32.

10. Mahonen M, Salomaa V, Brommels M, Molarius A, Miettinen H, Pyorala K, Tuomilehto J, Arstila M, Kaarsalo E, Ketonen M, Kuulasmaa K, Lehto S, Mustaniemi H, Niemela M, Palomaki P, Torppa J, Vuorenmaa T. The validity of hospital discharge register data on coronary heart disease in Finland Eur J Epidemiol 1997;13(4):403-15.

11. Hammar N, Larsen FF, de Faire U. Are geographical differences and time trends in myocardial infarction incidence in Sweden real? Validity of hospital discharge diagnoses.J Clin Epidemiol 1994;47(6):685-93.

12. Rosamond WD, Chambless LE, Sorlie PD, Bell EM, Weitzman S, Smith JC, Folsom AR. Trends in the sensitivity, positive predictive value, false-positive rate, and comparability ratio of hospital discharge diagnosis codes for acute myocardial infarction in four US communities, 1987-2000. Am J Epidemiol. 2004;160(12):1137-46.

13. McGovern PG, Jacobs DR Jr, Shahar E, Arnett DK, Folsom AR, Blackburn H, Luepker RV. Trends in acute coronary heart disease mortality, morbidity, and medical care from 1985 through 1997: the Minnesota heart survey. Circulation. 2001;104(1):19-24.

14. Rothman KJ, Greenland S. Introduction to categorical statistics. Person-time data: large-sample methods. Pure count data: largesample methods. In: Modern Epidemiology. 2nd ed. Philadelphia: Lippincott Williams and Wilkins; 1998.

15. Mantel N, Haenszel WH. Statistical aspects of the analysis of data from retrospective studies of disease. J Natl Cancer Inst 1959;22:719-48.

16. Brameld KJ, Holman CD, Lawrence DM, Hobbs MS. Improved methods for estimating incidence from linked hospital morbidity data. Int J Epidemiol. 2003;32(4):617-24.

17. Rosen M, Alfredsson L, Hammar N, Kahan T, Spetz CL, Ysberg AS. Attack rate, mortality and case fatality for acute myocardial infarction in Sweden during 1987-95. Results from the national AMI register in Sweden J Intern Med. 2000;248(2):159-64.

18. Rasmussen S, Abildstrom SZ, Rosen M, Madsen M. Case-fatality rates for myocardial infarction declined in Denmark and Sweden during 1987-1999. J Clin Epidemiol. 2004;57(6):638-46.

19. Abildstrom SZ, Rasmussen S, Rosen M, Madsen M. Trends in incidence and case fatality rates of acute myocardial infarction in Denmark and Sweden. Heart. 2003;89(5):507-11.

20. Hammar N, Alfredsson L, Rosen M, Spetz CL, Kahan T, Ysberg AS. A national record linkage to study acute myocardial infarction incidence and case fatality in Sweden. Int $\mathrm{J}$ Epidemiol 2001;30 Suppl 1:S30-4.

21. Pajunen P, Paakkonen R, Juolevi A, Hamalainen H, Keskimaki I, Laatikainen T, Moltchanov V, Niemi M, Rintanen H, Salomaa V. Trends in fatal and non-fatal coronary heart disease events in Finland during 1991-2001. Scand Cardiovasc J 2004;38(6):340-4. 